\title{
Target loading from a submerged explosion
}

\author{
Andrew Wardlaw ${ }^{\mathrm{a}, *}$, William Fourney ${ }^{\mathrm{b}}$ and Ulrich Leiste ${ }^{\mathrm{b}}$ \\ advanced Technology Research, Colombia, MD, USA \\ ${ }^{\mathrm{b}}$ University of Maryland, College Park, MD, USA
}

Received 3 March 2009

Revised 20 August 2009

\begin{abstract}
The pressure on a flat plate suspended over a submerged detonation is measured and simulated. Calculation and experiment are in relatively good agreement, although there is variation in experimental results and simulations are sensitive, near the centerline, to the computational details. This sensitivity is linked to the instability of the accelerating plume, typical of a Richtmyer-Meshkov instability. The plate loading features an initial force at plate center, followed by an expanding circular loading pattern. The initial load is due to plume impact, while the circular load arises from the impact of water transported up the edges of the explosion cavity.
\end{abstract}

\section{Introduction}

This paper describes pressures on a plate suspended above a detonation submerged in water. It examines mechanisms operative over a range of plate stand-offs and charge depths. This problem is of interest because it is similar to that of a buried charge in saturated soil and thus it is associated with landmine simulation. Recent experiments [1] indicate that plate pressures from a submerged charge are similar to that for a buried one. Figure 1, for example, demonstrates the similarity of the peak pressure distribution on a plate suspended over a submerged charge as opposed to a buried one. Compared to the buried explosive problem, the submerged explosive case is attractive from a computational point of view; it replaces sand, which is difficult to simulate and resists shear, with water, whose properties are well known. Studying this problem allows numerical uncertainties to be separated from sand modeling issues. While there have been extensive experimental and computational studies of buried explosives (e.g. [2-4]), little work appears to have been done on submerged charges; previous work by the authors is the exception [5].

This paper validates the submerged explosive simulations by comparing them to the measurement made by Fourney and Leister [1]. Measured and computed pressures are compared at the plate center and at several other stand-offs. Once validated, the computational model is applied to a variety of charge depths and plate stand-offs.

\section{Experiment}

The experimental setup is shown in Fig. 2 [1]. An aluminum target block, $25.4 \mathrm{~cm}$ square and $5.08 \mathrm{~cm}$ thick, is rigidly held $4 \mathrm{~cm}$ above the surface of a $1.5 \mathrm{~m}$ square, steel tank. The tank contains approximately $16.25 \mathrm{~cm}$ of water over a $6.35 \mathrm{~cm}$ layer of sand and a $5 \mathrm{~cm}$ thick gravel bed, which rests on the bottom of the tank. The explosive is 6.98 $\mathrm{g}$ of Detasheet submerged to a depth of $1 \mathrm{~cm}$ and initiated by an RP 87. The latter device is a detonator containing 69 gms of explosive within a thin metal casing. The Detasheet plus RP 87 is estimated to be the equivalent of $4.4 \mathrm{~g}$ of PETN (Pentaerythritol tetranitrate).

\footnotetext{
${ }^{*}$ Corresponding author. E-mail: awardlaw @atrcorp.com.
} 


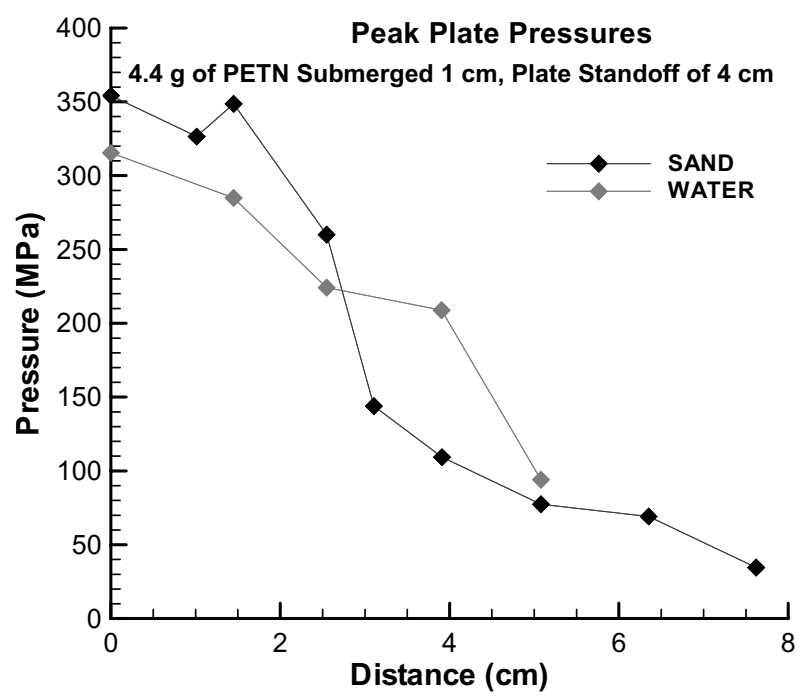

Fig. 1. Water and saturated sand pressure peaks.

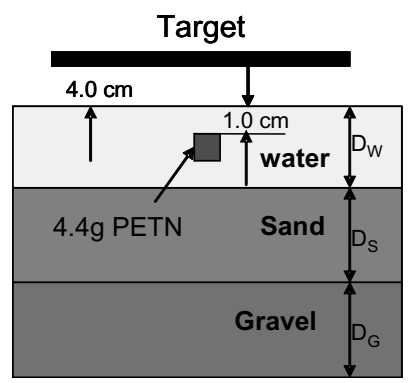

Fig. 2. Experimental setup.

Pressures are measured using $0.635 \mathrm{~cm}$ in diameter steel Kolsky bars. Each bar extends through a hole in the aluminum block and is positioned with its face flush with the plate lower surface. A bushing between the rod and the edges of the plate hole prevents water from penetrating the plate surface, while still permitting relatively free bar motion. Strain gages, located $30 \mathrm{~cm}$ up the bar, measure the resulting strain which is converted into pressure. The valid measurement window for the Kolsky bar is limited to $0.4 \mathrm{~ms}$, at which time the strain wave induced by water impact on the bar face reflects off the end of the rod and returns to the strain gage location.

\section{Computational methodolgy}

Experiments were simulated using the DYSMAS [6] (DYnamic System Mechanics Advanced Simulation). This code is designed to treat the explosive loading of a submerged structure and consists of a fluid and structural module that can be run in parallel. The fluid module, based on a second order Godunov scheme, provides the methods of simulation; the aluminum target block does not significantly deform and is treated as rigid, negating the need for the structural component. This fluid code treats multiple materials and tracks individual specie mass and energy. A single pressure and velocity is defined in each cell, even those containing multiple materials. Experimental pressure data is limited to $0.4 \mathrm{~ms}$, which permitted short simulations that terminated before the block edges influenced the solution. This allowed all simulations to be done in 2-D axisymmetric coordinates.

The computation replicates the setup shown in Fig. 2. Water is modeled using a polynomial equation of state [7], air is represented by a gamma law equation of state, with $\gamma=1.4$, and the saturated sand is simulated using a Tait 

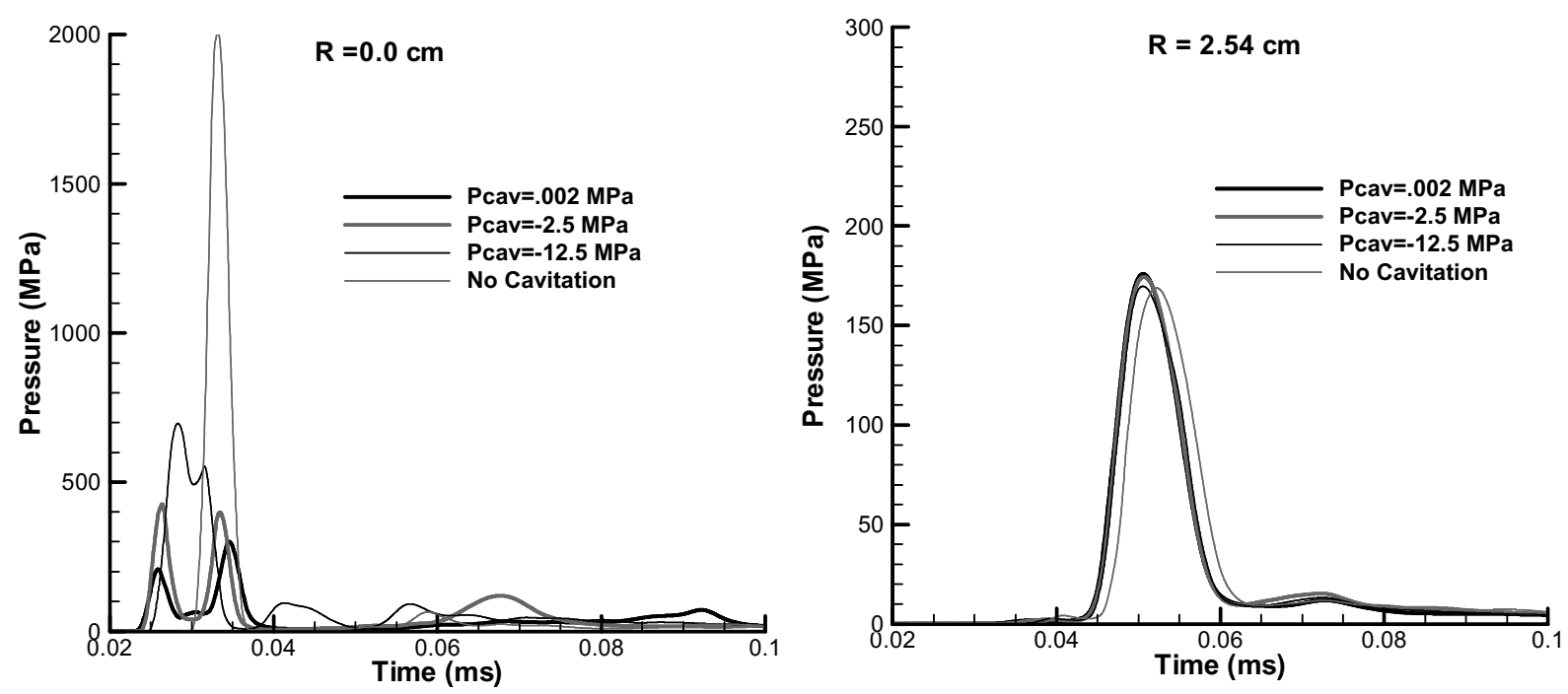

Fig. 3. Effect of cavitation on surface pressure.

formulation, where pressure is solely a function of density. A JWL (Jones-Wilkins-Lee) equation of state is used to model the explosive.

The explosive was simulated with $4.4 \mathrm{~g}$ of PETN, which is the explosive content of the Detasheet and detonator. In the simulation it was ignited instantaneously at the start of the problem (i.e. constant volume detonation). This method of modeling introduces the correct energy into the flow field but at a lower pressure than would a burn model. This type of detonation modeling is reasonable for this experiment; the small explosive size and the inert materials in Detasheet make it unlikely that a high burn pressure would be achieved.

Calculations are done on four different grids, with minimum cell sizes of $0.08,0.04,0.02$ and $0.01 \mathrm{~cm}$. These will be referred to as grids 1 to 4 , with 1 being the coarsest and 4 being the finest. All grids are uniform in the vicinity of the charge and stretched at the periphery of the computational domain to reduce the problem size. The total number of cells varied from 82,000 on grid 1 to 4.2 million for grid 4.

Most of the calculations were completed using a CFL time step safety factor of 0.45 . The CFL (i.e. CourantFriedrichs-Lewy) condition defines the maximum permissible time step size for an explicit computational method. Physically, it represents the time required for an acoustic wave to cross the smallest element in the calculation. It is a necessary but not sufficient condition and hence is used with a safety factor, a number between zero and one. The smaller the safety factor, the smaller the time step.

DYSMAS has the capability of implementing a pressure floor cavitation model for water [6] that prohibits the pressure from dropping below a prescribed threshold. This model, which is conservative, is intended for bubbly water regions found in bulk cavitation zones, and allows water to expand rapidly once the cavitation threshold has been reached. This cavitation model has been successfully compared to free ocean explosive tests and found to successfully capture cavitation formation and collapse pressure [7]. It also accurately represents cavitation effects in an internal explosion [8].

The experimental pressure represents the average over the face of the Kolsky bar which is acquired using equipment with a response time of $500 \mathrm{KHz}$ and post-processed by smoothing over a $2 \mu$ s window. Before comparing calculation and experiment, it is necessary to spatially average predicted pressures over the gage area and filter the resulting pressure to account for the equipment response time and post-processing. Details of this procedure are given in [5].

\section{Computational results}

The effect of changes in grid sizes on predicted gage pressure has been studied. At the plate center, twin pressure peaks occur for Grids 3 and 4 but are resolved as a single peak on Grids 1 and 2. By contrast, at a $5.08 \mathrm{~cm}$ distance 


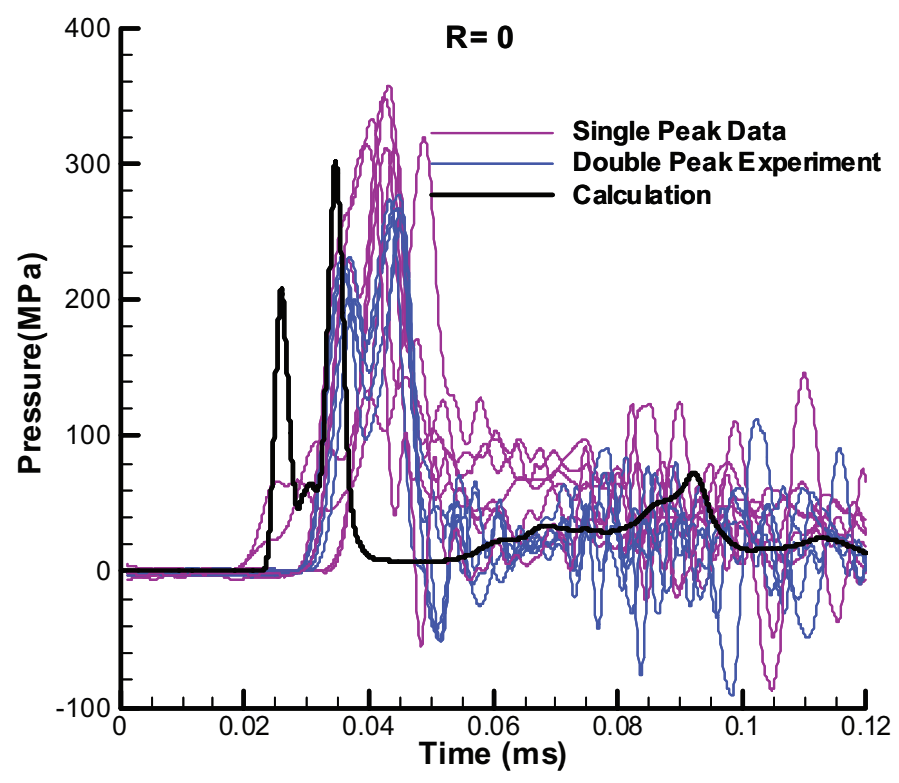

Fig. 4. All pressure data at $\mathrm{R}=0$.

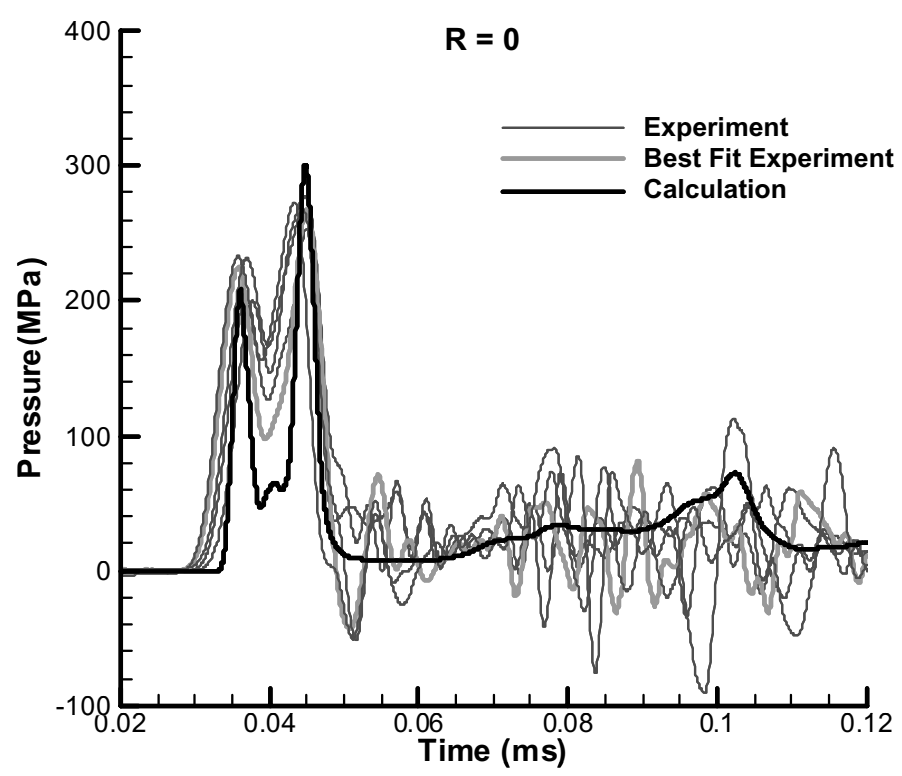

Fig. 5. Pressures at $\mathrm{R}=0$.

from the center of the plate, Grids 1 through 4 give similar results. The calculations presented in the remainder of this paper are done on Grid 3, which is the smallest grid that resolves the centerline dual pressure peaks.

The influence of the cavitation model on the surface pressures is shown in Fig. 3. At the centerline, its use is mandatory; otherwise pressures are too high and have a single peak instead of a double peak. Away from the centerline, the effect of cavitation is not very pronounced. Unless otherwise stated, a cavitation pressure of 0.002 MPa will be used, which is the default value applied in previous work [7,8].

Comparison of predicted and measured pressures is problematic - data from different tests show wide variations in peak pressures and functional form. The comparison procedure used here is to find a set of experimental curves that are similar in form to the predicted curve and focus on these results. As an example, the computed and measured 


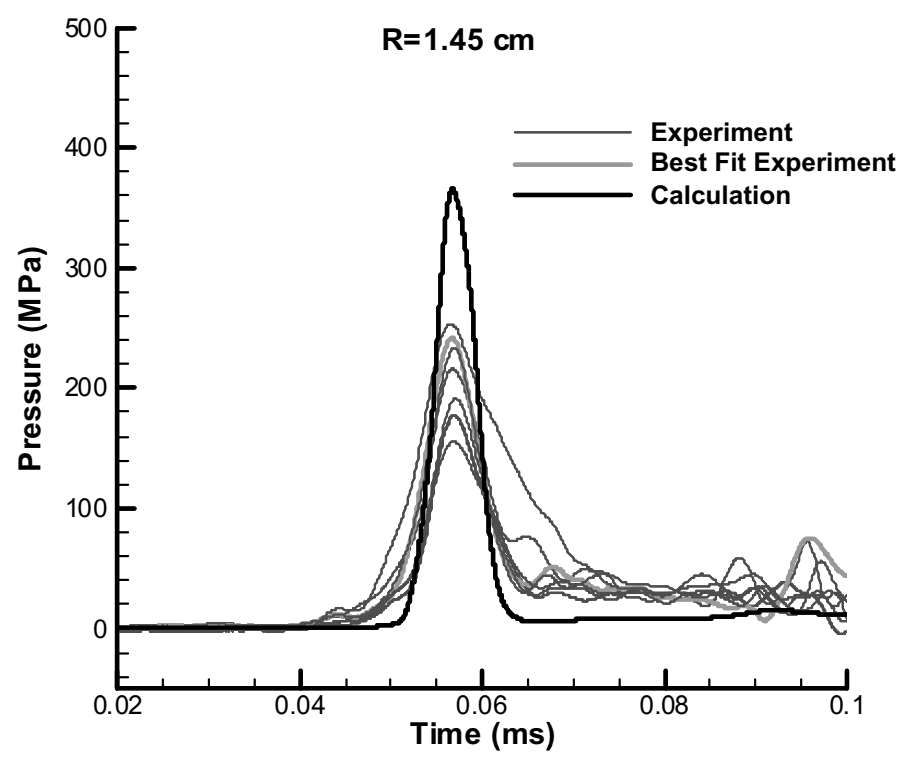

Fig. 6. All pressure data at $\mathrm{R}=1.45 \mathrm{~cm}$.

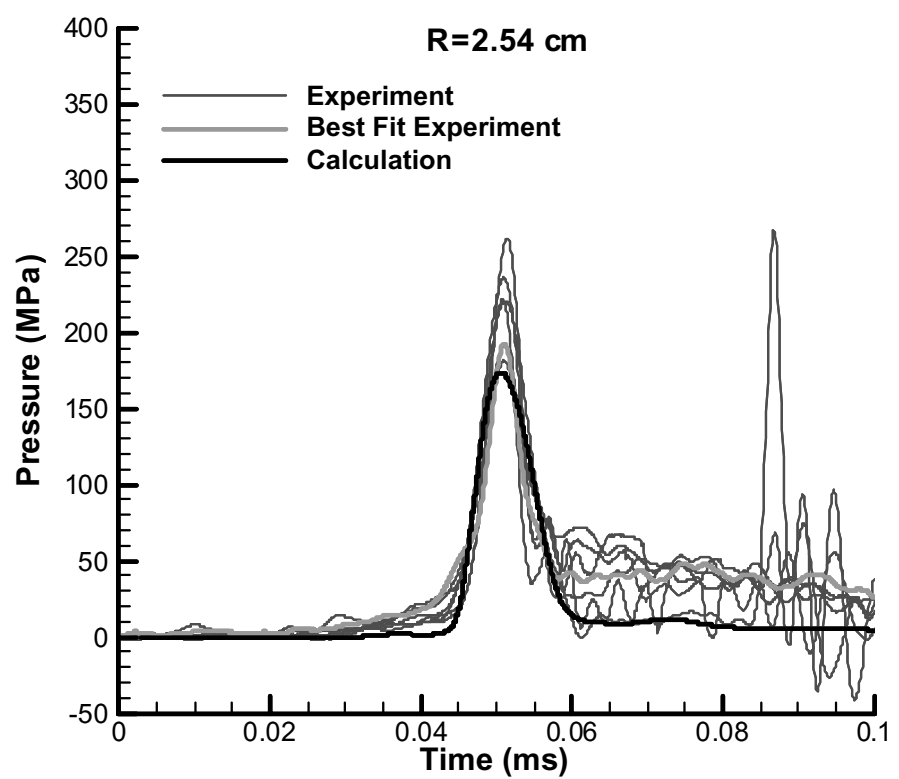

Fig. 7. Pressures at $\mathrm{R}=2.54 \mathrm{~cm}$.

centerline pressure histories are shown in Fig. 4. The predicted curve is in black, while the measured pressure histories are in blue and purple. The blue curves are similar to the calculated results; they have two peaks and similar peak values. By contrast, the purple curves have a single and often higher peak pressure. Removing the purple curves from the figure and translating the predicted pressure history in time to match the initial experimental pressure peak produces Fig. 5, which serves as a basis for discussion.

The scatter in experimental data shown in Fig. 4 is likely caused by the irregular surface of the water plume. Photos of such a plume show protruding fingers [9], which likely cause variations in surface pressure. In the case of the center gage, small discrepancies in position may significantly alter the pressure history. It is plausible that only a small misalignment of the center gage and charge center could produce a single rather than a double peaked 


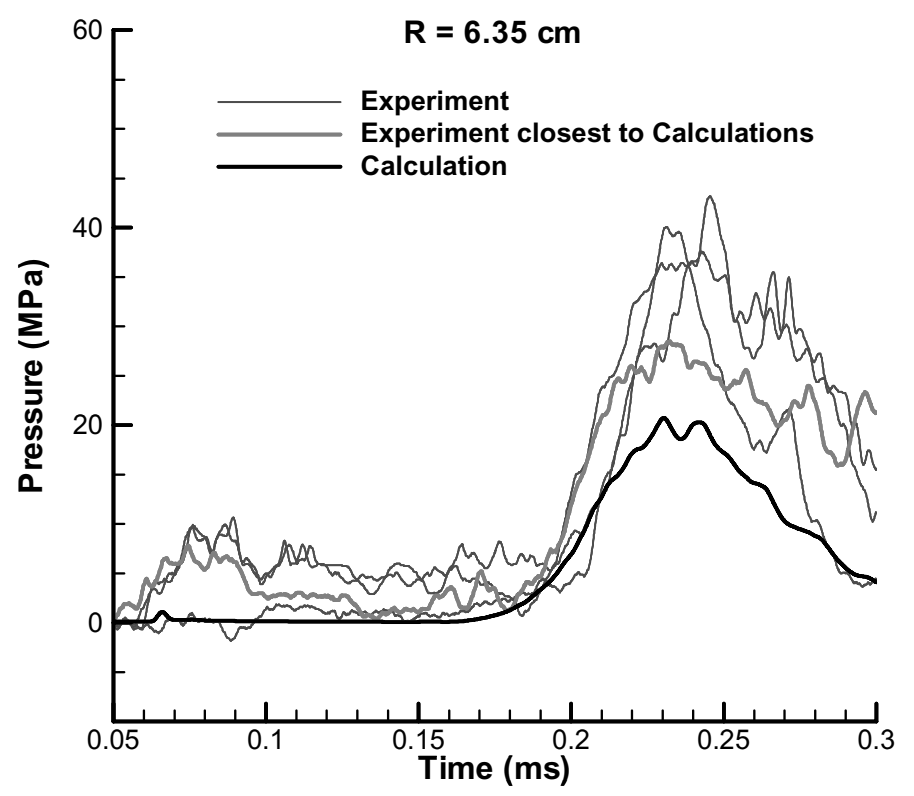

Fig. 8. Pressures at $\mathrm{R}=6.35 \mathrm{~cm}$.

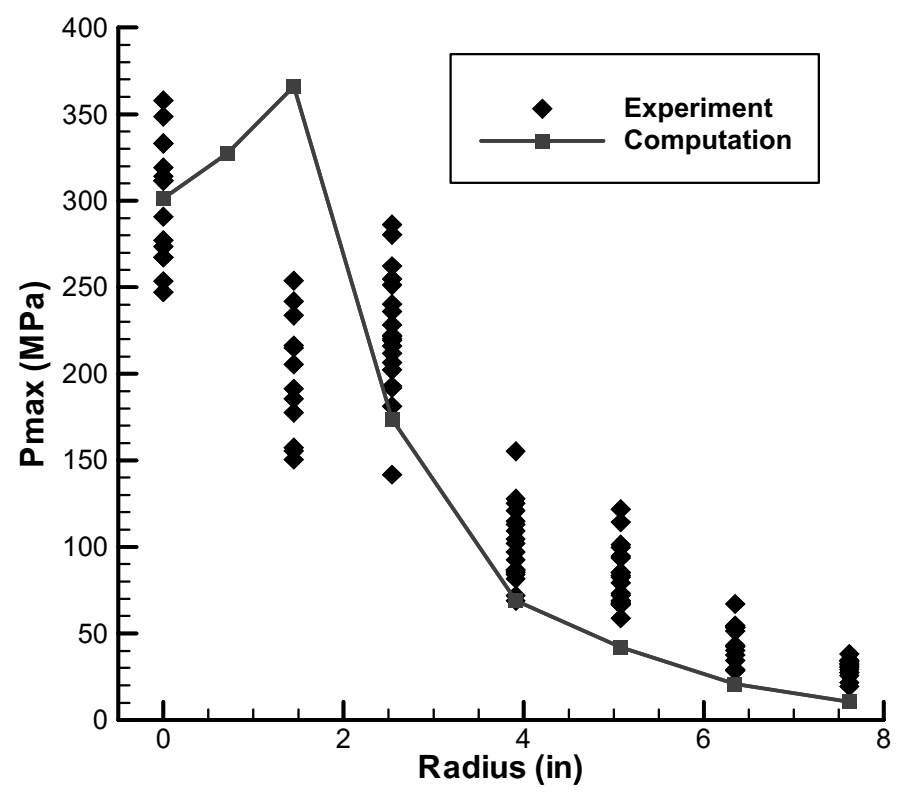

Fig. 9. Peak pressures.

pressure curve.

A comparison of predicted and measured surface pressure histories off the center line is shown in Figs 6 to 8 . Here experimental and predicted curves are altered as in Fig. 5. These figures show reasonable agreement between experiment and calculation with respect to functional form and pulse width. However, the calculated peak pressure tends to be lower than the experimental one far from the plate center.

Predicted and measured peak pressures are compared in Fig. 9. Here it can be seen that predictions reflect the magnitude of the measured values and decrease with radius as seen in experiment. However, experimentally there is a local minimum near $1 \mathrm{~cm}$ that is not captured by the calculation. 

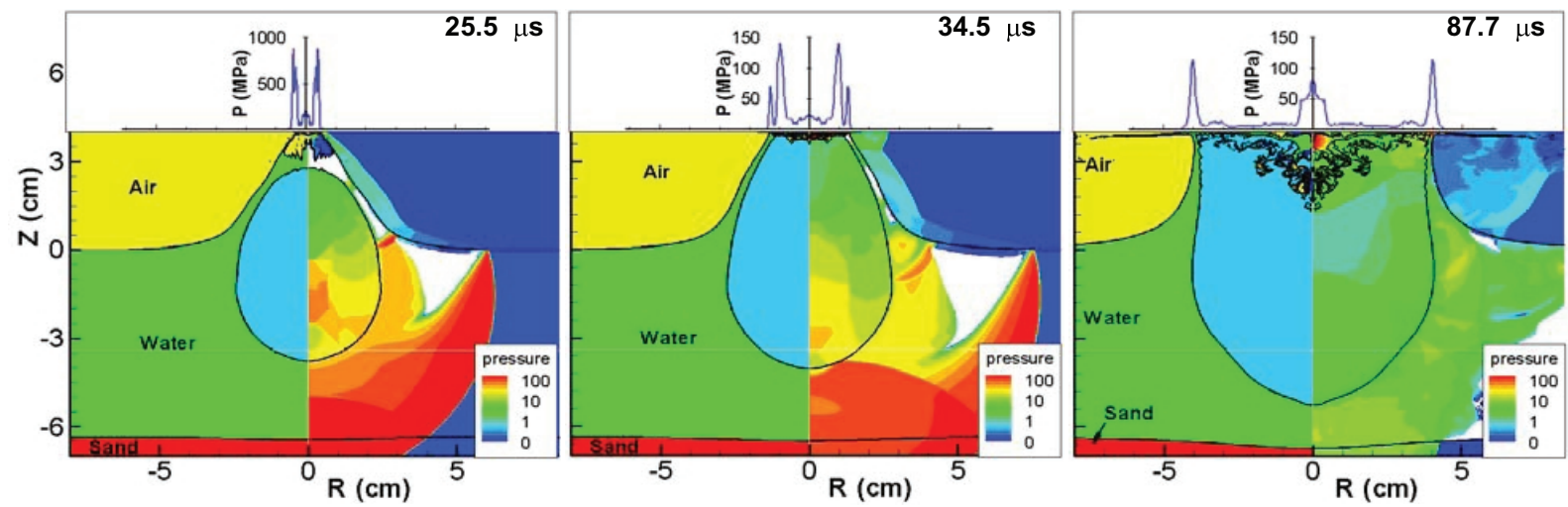

Fig. 10. Flow field development for a charge depth of $1 \mathrm{~cm}$ and plate sand-off of $4 \mathrm{~cm}$.

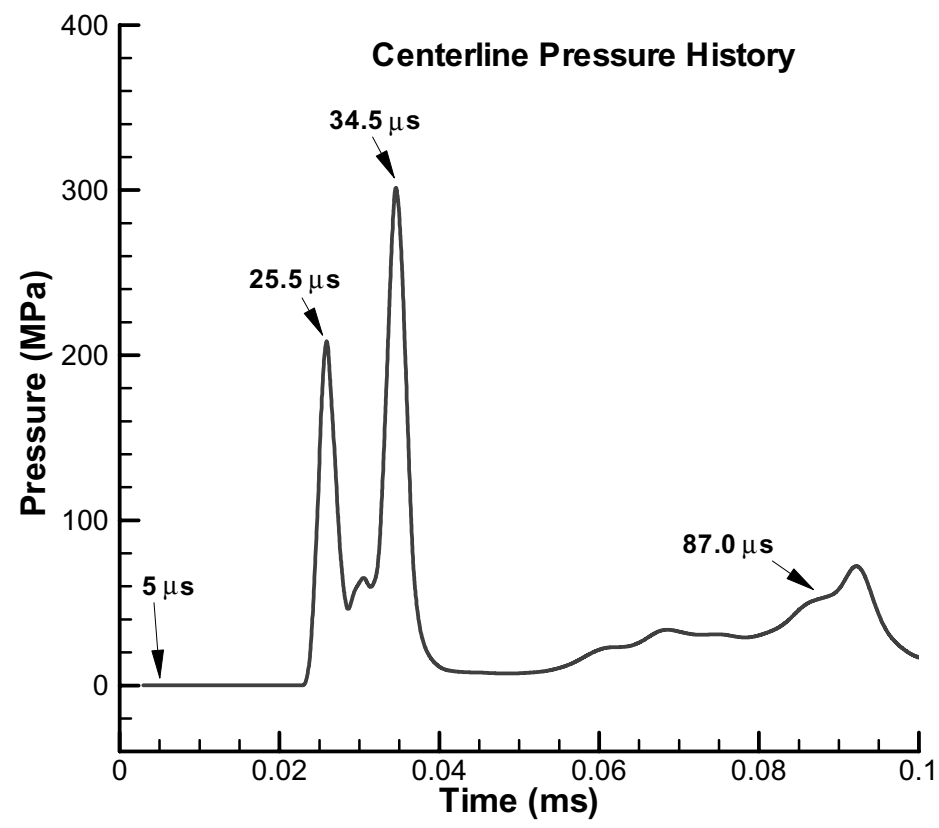

Fig. 11. Centerline pressure water first meet at the center of the plate.

\section{Analysis of results}

The simulated flow field is depicted in Fig. 10. The left side of each figure provides the material distribution while the right displays the pressure contours in $\mathrm{MPa}$, with the white regions representing cavitated flow. The top line graph gives the predicted pressure profile along the bottom of the block. Shortly after charge detonation, the shock reaches the water surface, accelerating it upwards. The shock reflects off the surface as an expansion, reducing the pressure in the plume (i.e. between the surface and the explosion bubble) and causing it to cavitate. The plume, propelled by the expanding gas bubble, continues to rise at supersonic velocity relative to the air. An air shock can be seen to precede it (see light blue region on the right side of Fig. 10 at $25.5 \mu$ s and $34.5 \mu \mathrm{s}$ ). Much of this plume remains cavitated during its upward travel.

The centerline pressure trace shown in Fig. 11 is indicative of the structure of the plume that strikes the plate.

The plume strikes at about $25 \mu \mathrm{s}$, producing the first pressure peak shown in this figure. The pressure then falls as the cavitated portion of the plume material reaches the plate. The second pressure peak occurs at $34.5 \mu$ s and represents an interaction between the reflected shock off the plate and the upward moving water in the immediate 

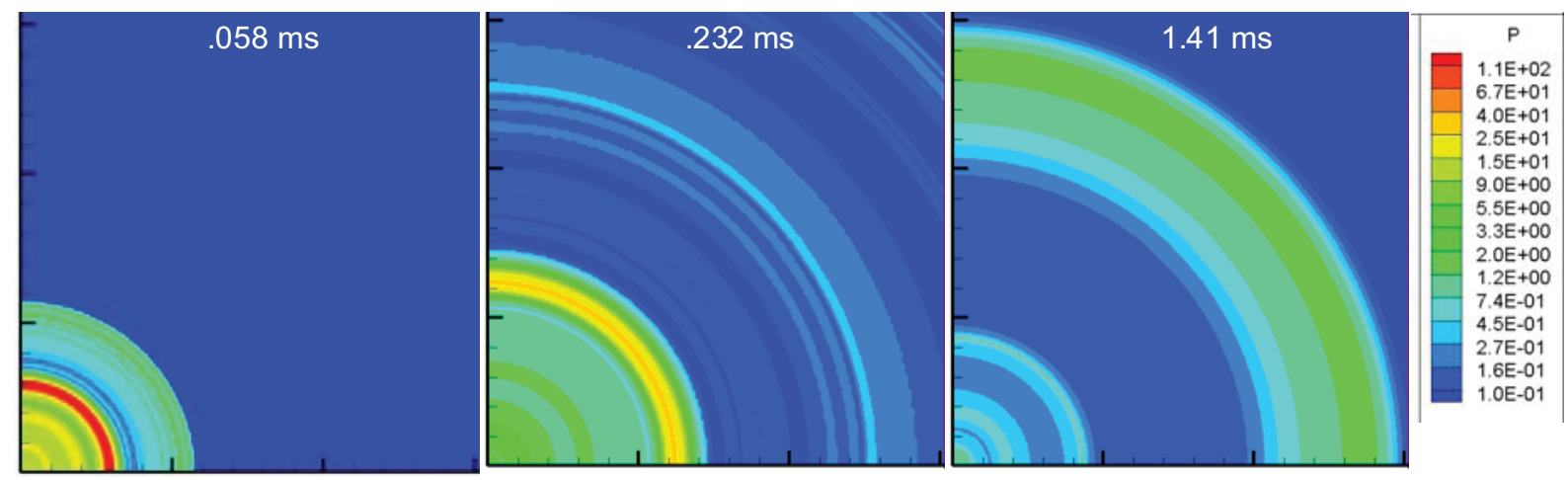

Fig. 12. Plate surface pressure distribution (MPa).

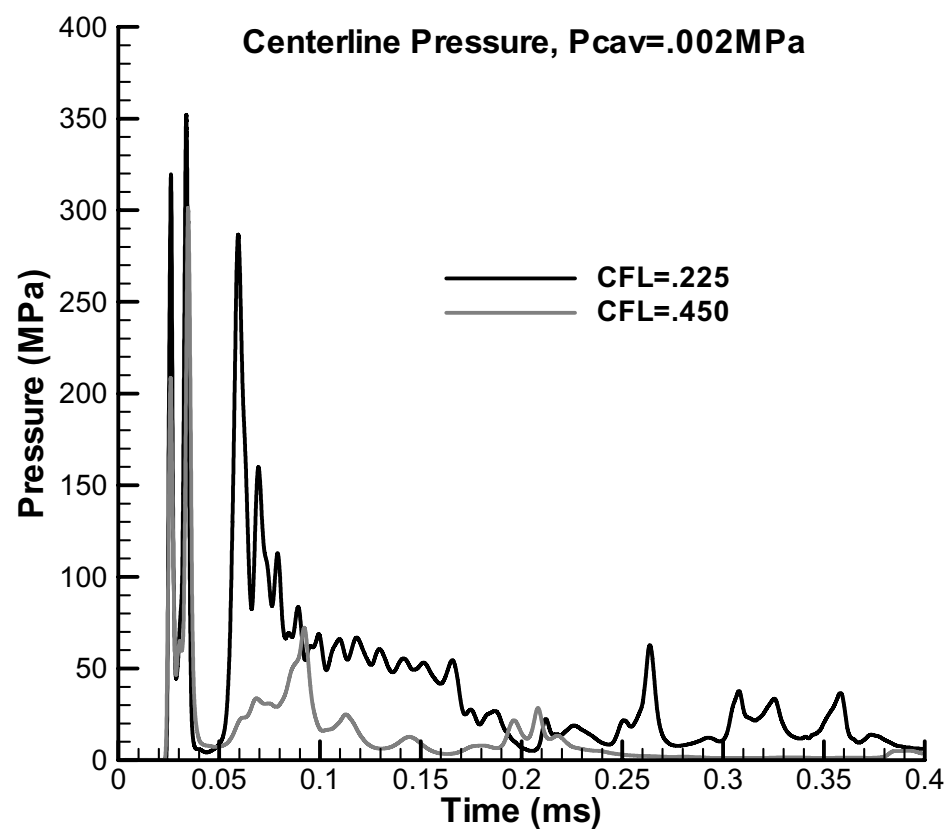

Fig. 13. Influence of step size on centerline pressure.

vicinity of the bubble surface. At this point the explosion bubble is sealed from the atmosphere by the surrounding water edges visible in Fig. 10. Water continues to be pumped up against the plate through the edges of the bubble. This stream divides when it hits the plate; part moves inward and part outward toward the edge of the plate. The water moving inward converges to form a downwards jet. The third pressure peak in Fig. 11, at $87 \mu$ s, occurs as the converging streams of water first meet at the center of the plate.

The plate is initially loaded at its center, but this load abates quickly and, as shown in Fig. 10, is replaced by a load that peaks above the edges of the bubble. The edges of the bubble are a conduit for high speed water that impacts the plate and accounts for this loading pattern. This leads to an expanding circle of high pressure whose peak value diminishes and whose pulse width widens in time. The plate loading is shown in Fig. 12 at several different times.

The calculations discussed in the last two sections have been completed using a time step CFL safety factor of 0.45 . This calculation has been repeated with the safety factor reduced to 0.225 , which should produce nearly identical results. While this occurs for most of the flow field, along the centerline, the jet formation pressure (i.e. the third peak) differs both in magnitude and time of initiation, as shown in Fig. 13. The reason for this can be seen by examining the condition of the water plume predicted in the two simulations, shown in Fig. 14. At the larger step 


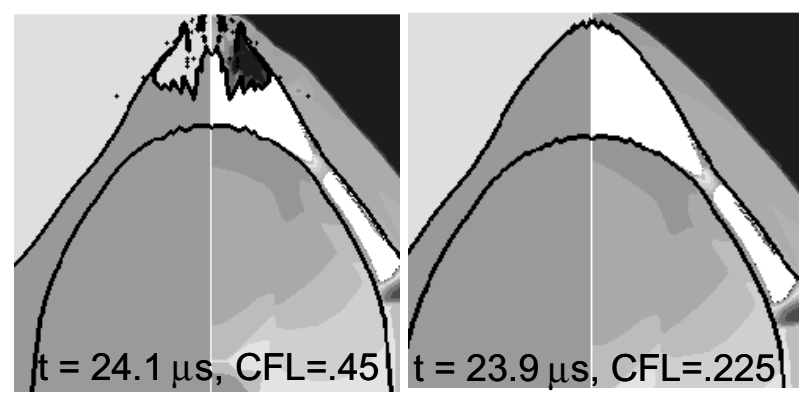

Fig. 14. Plume prior to striking plate.

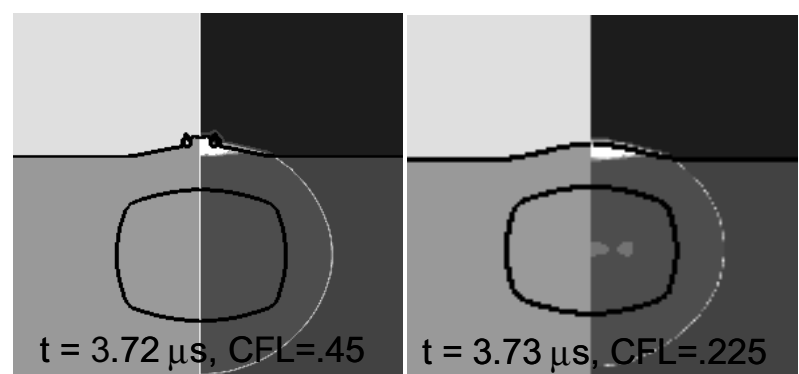

Fig. 15. Plume shortly after shock arrival.

size, the upper surface of the plume becomes irregular, while at the smaller step size, it remains fairly smooth. This irregularity reduces the first two pressure peaks, but more importantly, it changes the profile of the material striking the plate from an air-water mixture to water alone. In the latter case, it takes longer for the inward stream to form into a jet and when it does, it occurs at a lower pressure.

The difference in the computed plume geometry, shown in Fig. 14, is initiated when the detonation shock reaches the water surface. In Fig. 15, it is evident that at the larger step size, the shock creates a small disturbance which is absent at the smaller step size. Presumably the calculation at the smaller step size is slightly more dissipative which suppresses this disturbance. The sensitivity of the computed plume surface may be related to the well-known Richtmyer-Meshkov instability that occurs when an interface between fluids of differing density is impulsively accelerated.

\section{Other charge depths and plate stand-off conditions}

Additional calculations have been performed covering a range of plate stand-offs and charge depths. Figures 16 to 18 illustrate the flow field development for three of these cases and also provide centerline pressure histories. As with previous flow field illustrations, the left side of each figure is the material distribution and the right the pressure contours with white representing cavitated regions. The cases presented are a deeply submerged charge with the plate above the surface, submerged charge with plate on the surface, and surface charge with the plate above the surface. As long as the plate is located above the water surface and the charge is submerged, the flow evolution is similar to that discussed above. However, for different charge depth and plate stand-offs, the details differ, as do the timing of the various events. If the plate or charge is positioned on the water surface, different flow features occur and the detonation shock strikes the plate directly.

Figure 16 illustrates a deeply submerged charge with the plate located above the surface. Here the plume hits the target at about $55 \mu$ s producing the first pressure peak shown in this figure. The plume impact generates a reflected shock that travels towards the bubble and collides with the non-cavitated, upward moving water outside the expanding bubble. This creates a high pressure region in the flow field and the second pressure peak. Additionally, the high pressure region helps collapse the cavitated region. 

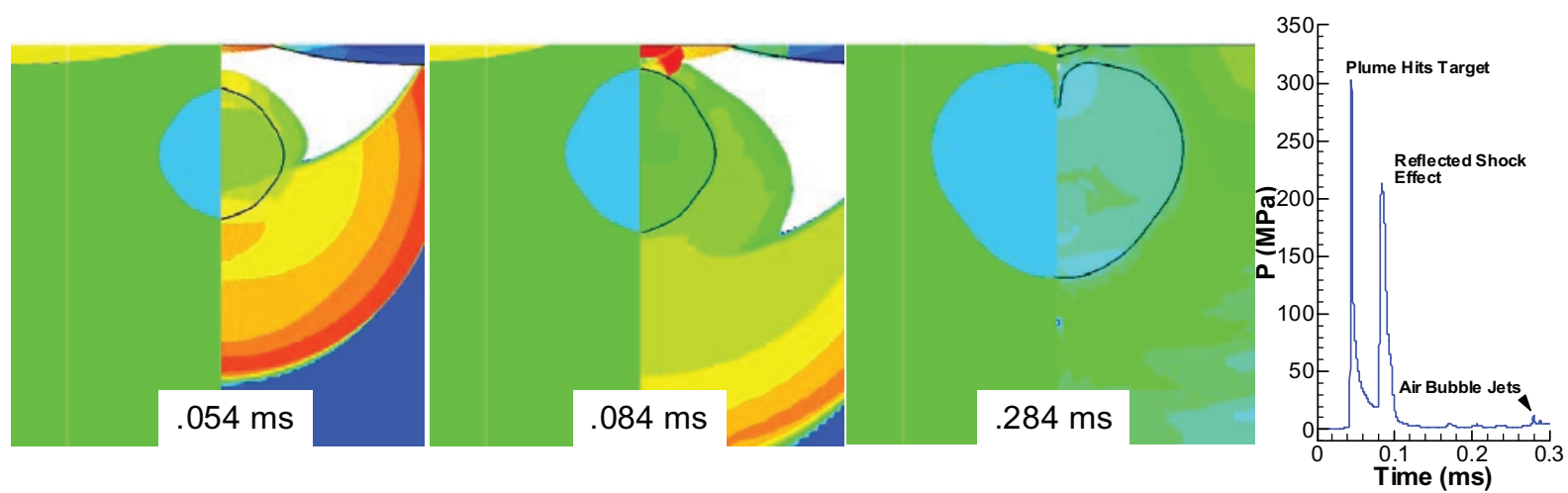

Fig. 16. Flow field for a stand-off of $1 \mathrm{~cm}$ and depth of $4 \mathrm{~cm}$.

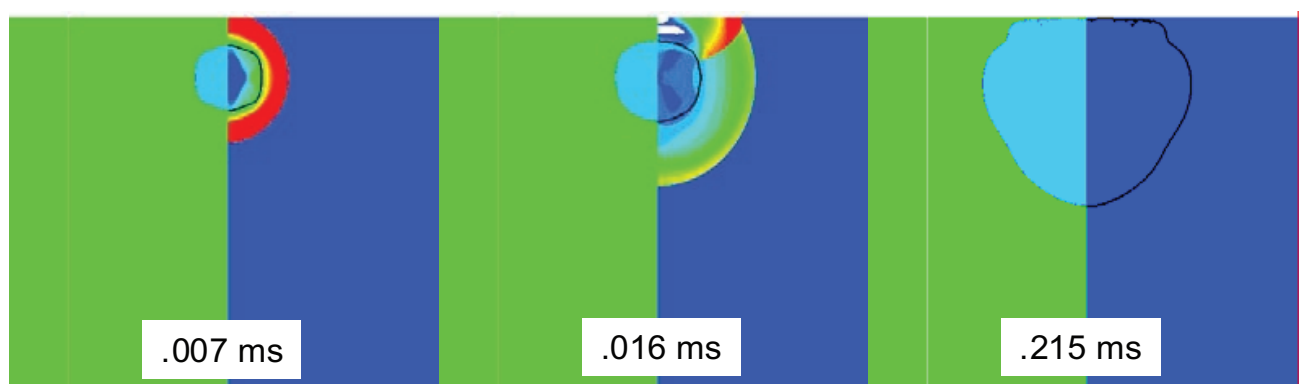

Fig. 17. Flow field for a surface plate and depth of $2 \mathrm{~cm}$.
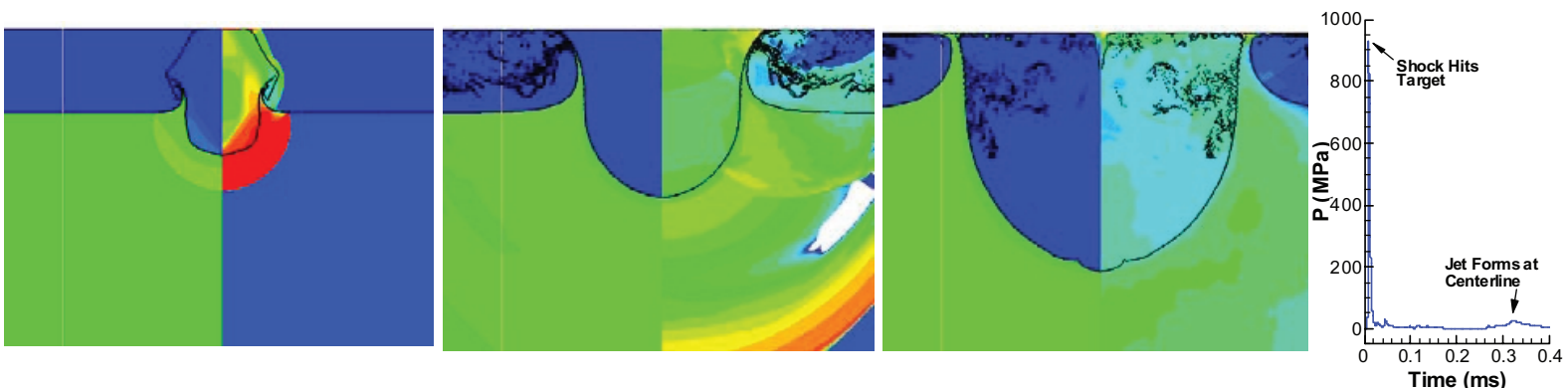

Fig. 18. Flow field for a surface charge and plate stand-off of $4 \mathrm{~cm}$.

Unlike the case studied earlier in this paper that features a shallower charge, the plume upper surface is relatively flat and smooth. It traps a pocket of air near the center of the plate that expands as the pressure decreases at the plate center and may contribute to the formation of the centerline jet shown at $0.284 \mathrm{~ms}$ in Fig. 16 .

A case featuring a plate located on the water surface and subjected to a charge submerged to a depth of $2 \mathrm{~cm}$ is shown in Fig. 17. Here the primary loading is from the detonation shock, not plume impact. The shock has a direct, single material path to the target, generating the high peak pressures, shown in Fig. 17, that greatly exceed those in which the plume impact is the dominant loading mechanism. The shock that strikes the plate reflects off the plate as a shock and then off the explosion bubble as an expansion, with low enough pressures to induce cavitation. Later, as can be seen if Fig. 17, a small pressure pulse due to cavitation collapse occurs at about $0.05 \mathrm{~ms}$.

The final case considered is shown in Fig. 18 and consists of a surface charge located below a plate at a stand-off of $4 \mathrm{~cm}$. The shock from the explosive has a direct air path to the plate and that leads to the plate peak pressure 
in this figure. Although the charge is initially located at the water surface, water ejected by the explosion creates a sealed gas bubble beneath the plate that eventually forms a weak jet along the bubble centerline.

\section{Summary}

The detonation of a submerged charge beneath the plate suspended over a water surface has been investigated computationally using the DYSMAS code [6]. Simulations were completed for a range of plate stand-offs and charge depths and validated against laboratory scale experiments [1]. In these tests, pressures were measured using a Kolsky bar and results were obtained at a number of locations on the plate. Near the plate center predicted pressures are found to be sensitive to the cavitation model and an appropriate cavitation threshold was determined to be 0.002 $\mathrm{MPa}$, which is consistent with that used in previous studies [7,8]. Using this cavitation level, reasonably good agreement with experiment is achieved, both with respect to peak pressure values and pressure functional form.

The measured pressure history at the centerline in the validation case was difficult to repeat. The simulations provide insight as to the cause of this sensitivity; the shape of the water plume that strikes the plate tends to be unstable and is easily perturbed. Changes in the water plume shape affect the flow field evolution near the plate center and can influence the formation of a downward jet along the centerline.

The plate loading, as well as the centerline pressure history, depends on the charge depth and plate stand-off. For the typical case of a submerged charge and a plate suspended above the water, the loading is dominated by water ejected upwards by the detonation. The initial force on the plate occurs at its center and is caused by the plume strike. This event is then followed by a second peak created by the interaction of the shock reflected off the plate and the upward moving water in the vicinity of the expanding bubble. A downward moving jet can form along the centerline later in the calculation, leading in some cases to an additional pressure peak. Later in time, the plate loading is driven by edges of the explosion products bubble. Water is transported at high speed up these edges and impacts the plate, leading to an expanding circular peak plate pressure pattern.

The detonation shock has a direct, single material path to the plate in cases where either the plate or charge is on the surface. Here the shock produces pressures much larger than those associated with a plume strike. There is additional complexity to these flow fields and unexpected events can occur. The surface charge ejects enough water to form a sealed gas cavity below the plate while a plate located on the surface experiences secondary cavitation reloading.

This study is motivated by the experimental observation that the peak pressure distribution on a plate suspended over a submerged explosive is similar to that on a plate located above an explosive buried in saturated sand. The computational study of the buried, saturated sand case [10] also predicts similar plate loading mechanisms as reported in this paper. As with the submerged explosion, the buried explosion produces an initial pressure peak at the plate center due the plume strike, followed by a circular loading pattern induced above the edges of the explosion bubble. These similarities suggest that submerged explosions studies, using a well known water equation of state, provide a method of assessing the computational framework for buried explosive calculations without the uncertainty associated with sand characterization.

\section{Acknowledgement}

This work was supported by the U. S. Army Research Development, and Engineering Command, TARDEC and the Office of Naval Research - Expeditionary Warfare and Combating Terrorism Department. Points of contact were David Fox, TARDEV, Lee Mastroianni, ONR and Maj. Bill Short, USMC. The authors also wish to thank Mr. Timothy Hennessey of NSWC, Indian Head Division for his support of this effort.

\section{References}

[1] W.L. Fourney and U. Leiste, Unpublished Results from the Dynamics Effects Laboratory, University of Maryland, 2008.

[2] I. Kerley, Gerald, Numerical Modeling of Buried Mine Explosions, ARL-CR-461, March 2001. 
[3] L. Donahue, A. Bouamoul and T.E. Dunbar, Numerical Modeling Approaches for Simulation of Landmine Blast Loading, Proceedings of the 76th Shock and Vibration Symposium, Destin, Florida, October 30-November 3, 2005.

[4] M. Grujicic, B. Pandurangan, J.D. Summers, B.A. Cheeseman and W.N. Roy, Application of the Modified Compaction Material Model to Soil With Various Degrees of Water Saturation Shock and Vibration Journal 15 (2008), 79-99.

[5] A.B. Wardlaw, W.L. Fourney and U. Leister, Target Loading from a Submerged Explosive, Journal of Blasting and Fragmentation 2(3) (November 2008), 211-226.

[6] A.B. Wardlaw, Jr., J.A. Luton, J.R. Renzi, K.C. Kiddy and R.M. McKeown, The Gemini Euler Solver for the Simulation of Underwater Explosions, NSWC IH TR 2500, Nov. 2003.

[7] A. Wardlaw, Jr., R. Ilamin and G. Harris, Cavitation Modeling, Proceedings of the 73th Shock and Vibration Symposium, Nov. 2002.

[8] A. Wardlaw, Jr. and J.A. Luton, Fluid-Structure Interaction Mechanisms for Close-in Explosions, Shock and Vibration Journal 7 (2000), $265-275$.

[9] R.H. Cole, Underwater Explosions, Princeton University Press, Princeton, New Jersey, 1948, pp. 390-391.

[10] A. Wardlaw, S. Tidwell, T. Hennessey and W. Szymczak, Modeling Plate Loading Due to Buried Explosives, 9th Joint Classified Bombs/Warheads \& Ballistics Symposium", Aug. 2006. 

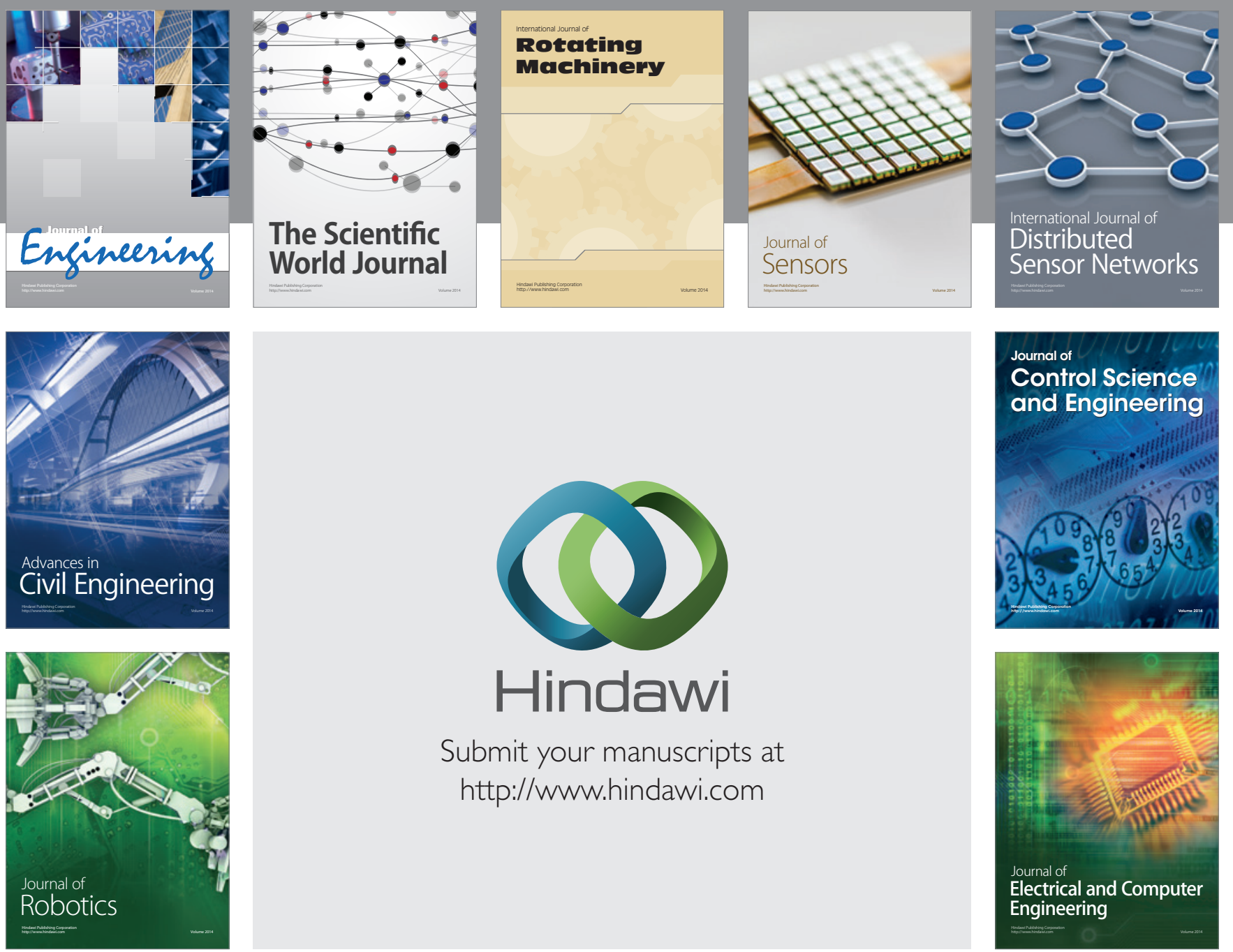

Submit your manuscripts at

http://www.hindawi.com
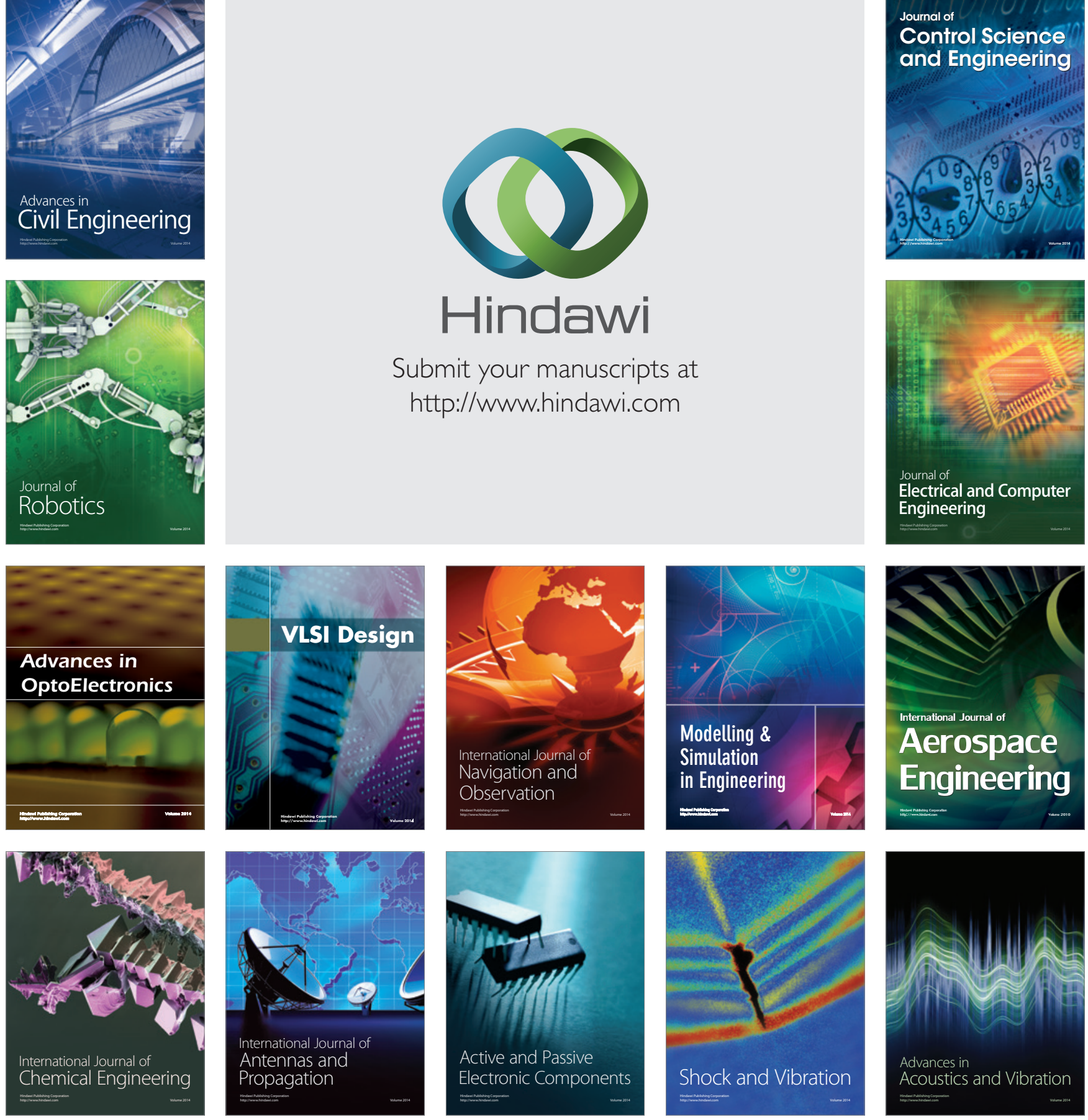